\title{
The Star Formation History of Leo I
}

\author{
C. Gallart and W.L. Freedman \\ Carnegie Observatories. 813 Santa Barbara St. Pasadena CA 91101, \\ $U S A$
}

\begin{abstract}
We advance some results of our study of the star formation history of the Local Group dSph galaxy Leo I, obtained through the analysis of its deep HST color-magnitude diagram (CMD) using model CMDs computed from stellar evolutionary models. We conclude that most star formation $(\simeq 90 \%)$ took place in Leo I between 7 and $1 \mathrm{Gyr}$ ago. It seems to have started at a very low rate $\simeq 15 \mathrm{Gyr}$ ago and continued, also at a very low rate, from 1 Gyr ago until the present time. A constant $\mathrm{Z}=0.0004$ and a large fraction of binary stars are required to obtain the best agreement with both the distribution of stars across the CMD and with its morphology. An IMF like the one obtained by Kroupa et al. (1993) for the solar neighborhood, or steeper, is compatible with the data.
\end{abstract}

\section{Introduction}

Leo I presents an enigmatic system with unique characteristics among Local Group galaxies. From its morphology and similarity to other dSph in terms of its lack of detectable quantities of H I (Knapp, Kerr \& Bowers 1978), it would be considered a dSph galaxy. With the exception of Leo I, an old stellar population traced by a horizontal-branch (HB) has been clearly observed in all the other $\mathrm{dSph}$ galaxy satellites of the Milky Way, regardless of their subsequent star formation histories (SFHs). In this respect, Leo I is an unusual galaxy which displays a well populated red-clump indicative of a large fraction of intermediateage stars, but no evident HB.

We have analyzed very deep F555W $(V)$ and F814W $(I) H S T$ data using model color-magnitude diagrams (CMDs) to obtain the SFH of Leo I. Because the data are deep enough to reach the magnitude expected for the oldest main sequence turnoffs, we are able to exploit our analysis method to the maximum of its capabilities. As a result, we have quantitatively obtained a SFH for Leo I with unprecedented detail, even for its early epoch of evolution.

\section{Observations}

WFPC2 HST $V(\mathrm{~F} 555 \mathrm{~W})$ and $I(\mathrm{~F} 814 \mathrm{~W})$ data in one $2.6^{\prime} \times 2.6^{\prime}$ field in the center of Leo I were obtained on March 5, 1994. Three deep exposures with both $V$ and $I$ filters (1900 s and $1600 \mathrm{~s}$ respectively) were taken. To ensure that the brightest stars were not saturated, one shallow exposure with each filter 
(350 $\mathrm{s}$ in $V$ and $300 \mathrm{~s}$ in $I$ ) was also obtained. Photometry of the Leo I stars was measured using the set of DAOPHOT II/ALLFRAME programs developed by Stetson (1994), and the final photometry on the Johnson-Cousins system was calibrated using the ground based photometry obtained by Lee et al. (1993).

In Fig. 1a we present the $\left[(V-I)_{0}, M_{I}\right]$ CMD for Leo I based on the three WF chips. It contains a total of 28000 stars with small photometric errors $(\sigma \leq 0.2)$. For a more complete description of the data reduction and a discussion of the features present in the CMD, see Gallart el al. (1999a; G99a hereafter).

\section{Computing the Star Formation History}

\subsection{Overview}

Our goal is to reconstruct the SFH of Leo I from the information in its CMD. The method we are using is based on the comparison of the distribution of stars in the observed CMD with that in the model CMDs resulting from a large number of possible SFHs. For our purposes, we consider the SFH to be a function of time defined by the star formation rate $\operatorname{SFR}(\mathrm{t})$, the chemical enrichment law $\mathrm{Z}(\mathrm{t})$, the initial mass function IMF and a function $\beta(f, q)$, controlling the fraction $f$ and mass ratio distribution $q$ of binary stars. We are analyzing large sets of models with different combinations of $\mathrm{Z}(\mathrm{t}), \mathrm{IMF}$ and $\beta(f, q)$. The comparison between the observed CMD and the model CMDs is done through $\chi^{2}$ minimization of the number of stars in a set of regions of the CMD, chosen to sample stars of different ages or in specific stellar evolutionary phases. For each set of Z(t), IMF and $\beta(f, q)$, a minimum of the $\chi^{2}$ function, which will provide the best fitting $\operatorname{SFR}(\mathrm{t})$ for that particular set of functions, is searched for. Whether this best fitting $\operatorname{SFR}(\mathrm{t})$ is a good (i.e. acceptable) $\operatorname{SFR}(\mathrm{t})$ is indicated by the value of $\chi^{2}$ itself. The details of this work and the definitive results will be presented in Gallart et al. (1999b, G99b hereafter). See also Aparicio (this conference) and Aparicio, Gallart \& Bertelli (1997) for a discussion of our synthetic CMD approach.

\subsection{The Parameter Space}

a) The IMF: We computed three sets of models with three different IMFs, with a common slope $m^{-1.3}$ for $0.08 \leq m<0.5$ as in Kroupa, Tout \& Gilmore (1993), and three different slopes for the upper mass interval, bracketing those of Kroupa et al.:

i) $m^{-3.2}$ for $0.5 \leq m<30$

ii) $m^{-2.2}$ for $0.5 \leq m<1.0 ; m^{-2.7}$ for $1.0 \leq m<30$

iii) $m^{-2.0}$ if $0.5 \leq m<30$

The stars in the CMD have masses above $M \simeq 0.6 M_{\odot}$, and therefore the star counts in the CMD do not depend on the slope of the lower mass segment. This low mass slope does affect the zero point of the star formation rate, and hence the total mass in stars and stellar remnants.

b) The chemical enrichment law: We calculated models with the following four metallicity laws:

i) Constant $\mathrm{Z}=0.0001$

ii) Constant $\mathrm{Z}=0.0004$ 
iii) Constant $\mathrm{Z}=0.0006$

iv) $\mathrm{Z}$ increasing with time from $\mathrm{Z}=0.0001$ to $\mathrm{Z}=0.0008$, with a Gaussian dispersion of $\sigma_{Z}=0.0001$ at each age. $\mathrm{Z}(\mathrm{t})$ has been determined by choosing the metallicity of an isochrone centered in the observed red-giant branch (RGB) for each age.

c) Binary fraction: In general, the two components of a binary star have different masses, and consequently different evolutionary status and color, and they may populate regions of the CMD that would not be populated by single stars. Therefore, it seems clear that binary stars may play an important role in the morphology and star counts across the CMD. The change in the star counts across the CMD will depend both on the fraction of binaries $(f)$ and on their mass-ratio $(q)$ distribution, both quite unknown quantities (Duquennoy \& Mayor 1991; Olszewski, Pryor \& Armandroff 1996).

In principle, only for mass ratios $q$ relatively close to unity (i.e., when the two components have similar masses) will the secondary have a substantial effect on the combined luminosity of the binary. For simplicity, in our models we have considered mass ratios in the interval $0.6 \leq q \leq 1.0$, with a few complementary tests using different $q$ intervals. We considered 5 different fractions of binary stars: $f=0,0.1,0.3,0.6$ and 0.9 .

\section{Results on the Star Formation History of Leo I}

The results of our search for a SFH that best reproduces the observed Leo I CMD yields the following conclusions:

- Most star formation $(\simeq 90 \%)$ took place in Leo I between 7 and 1 Gyr ago. Star formation seems to have started at a very low rate $\simeq 15 \mathrm{Gyr}$ ago and continued, also at a very low rate, from $1 \mathrm{Gyr}$ ago until the present time.

- A constant $\mathrm{Z}=0.0004( \pm 0.0001)$ provides the best agreement with both the distribution of stars across the CMD and with its morphology.

- A large fraction of binary stars $f$ provides the best fit.

- An IMF like the one obtained by Kroupa et al. (1993) for the solar neighborhood, or steeper, is compatible with the data.

Figure $1 \mathrm{~b}$ shows the model CMD obtained with the $\mathrm{Z}(\mathrm{t}), \mathrm{IMF}, \mathrm{SFR}(\mathrm{t})$ and $\beta(f, q)$ that provide the best solution for the SFH of Leo I. It can be compared with the observed CMD shown in panel (a) of the same Figure. The low value of $\chi^{2}$ for this model $\left(\chi^{2} \simeq 1\right)$ indicates the star counts across the CMD are closely matched by the model. Note, in addition, that most morphological details in the observed CMD are successfully reproduced, in particular:

- The extent of the main sequence to about $M_{I} \simeq 0$.

- The faint extension of the red-clump to the blue, i.e. to where the HB would be expected, and the gap between the brightest stars in the subgiantbranch and the remainder of the subgiant branch. This bridge of stars connecting the blue extension of the red-clump and the brightest main 


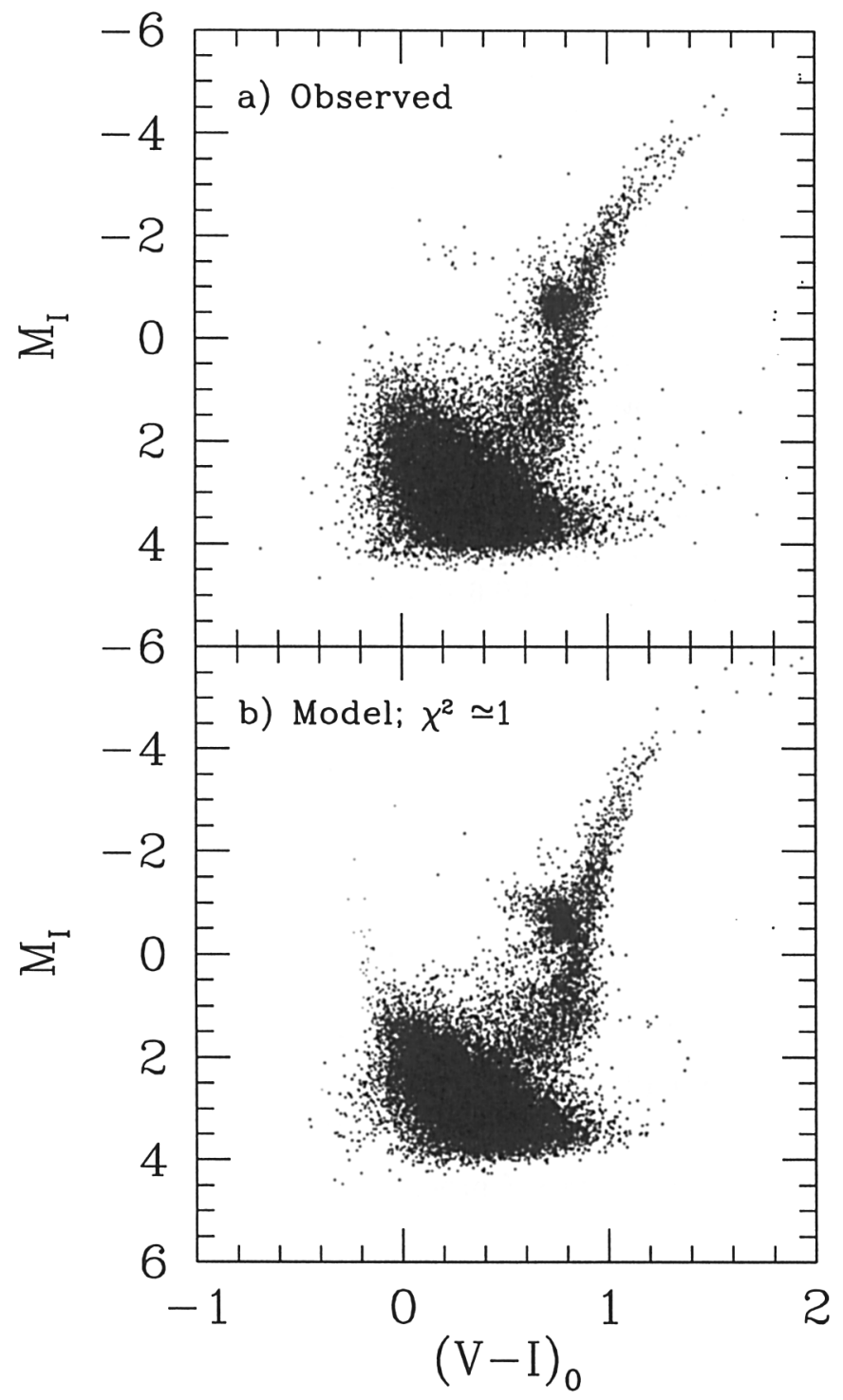

Figure 1. (a) Observed CMD of Leo I. (b) Best model CMD with IMF $m^{-3.2}(0.5 \leq m<30), \mathrm{Z}=0.0004$ and a large fraction of binary stars $f$ (see G99b for details). The value of $\chi^{2}$ indicates compatibility with the data. 


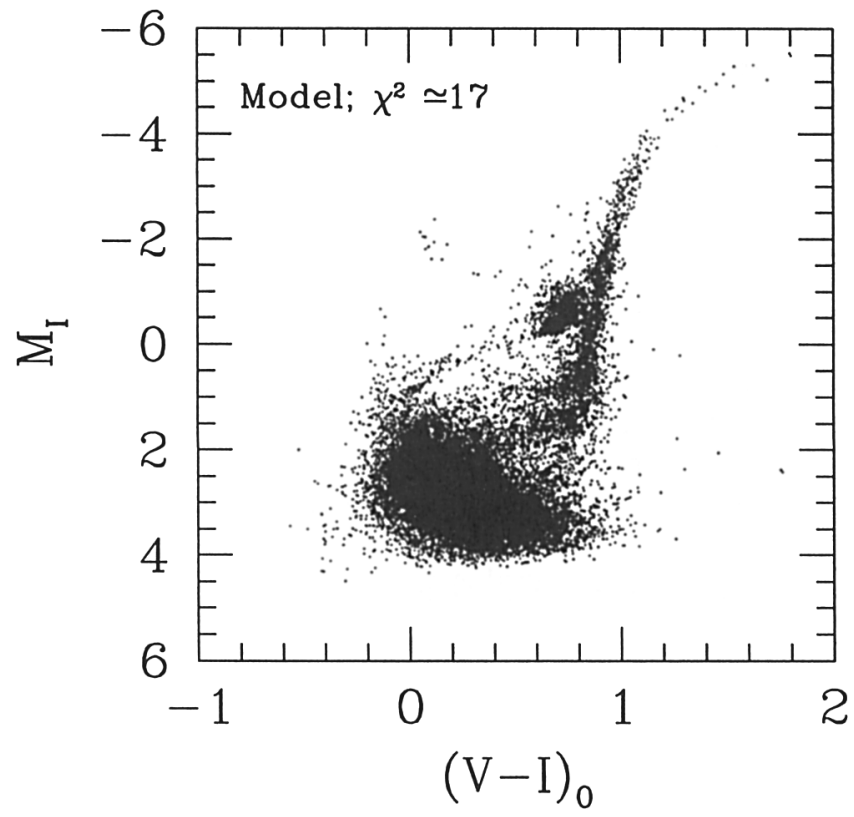

Figure 2. Best model CMD with IMF $m^{-2.0}(0.5 \leq m<30)$, $\mathrm{Z}=0.0001$ and a low fraction of binary stars (see G99b for details). The large value of $\chi^{2}$ indicates this model is incompatible with the data.

sequence turnoff can be explained mainly by $15-10$ Gyr old HB stars, plus some fraction of $1 \mathrm{Gyr}$ old subgiant stars. Therefore, it seems that the small fraction of old stars (less than 10\%) suggested by our models is indeed necessary to account for some morphological details of the observed CMD.

- The shape of the red-clump, and in particular, its elongation to brighter magnitudes. The different position angle in the shapes of the observed and model red-clump is probably related to the overall different inclination of the RGB between the model and the data, which is known to be due to the uncertainties in the transformation from the theoretical to the observational plane.

- The gradient in the density of stars across the subgiant branch, specially the step in density seen at $M_{I} \simeq 2$.

- The width of the RGB. It is interesting that only a Z constant with time produces an RGB width similar to the observations. If $\mathrm{Z}$ increases with time, even in a narrow interval, the RGB gets narrower. Therefore this supports the conclusion that $\mathrm{Z}$ must be constant, which is also obtained from the stellar counts across the CMD.

It is interesting to note that the general trend for $\mathrm{SFR}(\mathrm{t})$ is relatively similar for other models with sets of IMF, $\mathrm{Z}(\mathrm{t})$ and $f$ that result in large values of $\chi_{\nu}^{2}$. 
In other words, the result on SFR(t) is not strongly dependent on the IMF, $\mathrm{Z}(\mathrm{t})$ or $f$ (for reasonable choices of these parameters), but these parameters are the ones that determine whether a good agreement between the data and the models can be found.

Figure 2 shows the model CMD obtained from the best fitting $\operatorname{SFR}(\mathrm{t})$ in the case of an IMF $m^{-2.0}(0.5 \leq m<30), \mathrm{Z}=0.0001$ and a low $f$. This SFR(t) has similar characteristics to the previous one (i.e., most star formation occurs between 7 and 1 Gyr ago). Nevertheless, in this case the value of $\chi^{2}$ is high $\left(\chi^{2} \simeq 17\right)$. This indicates it is not possible to reproduce the star numbers across the CMD with any $\operatorname{SFR}(\mathrm{t})$ and this particular combination of $\mathrm{IMF}, \mathrm{Z}(\mathrm{t})$ and $f$. Note the substantial morphological differences between this CMD and the observed CMD in Fig. 1. This demonstrates we are able to distinguish between relatively similar SFHs.

Acknowledgements. C.G. acknowledge an IAU travel grant to attend this Symposium. Support for this work was provided by NASA grant GO-535003-93A from the Space Telescope Science Institute, which is operated by the Association of Universities for Research in Astronomy Inc. under NASA contract NASA5-26555.

\section{References}

Aparicio, A., these proceedings

Aparicio, A., Gallart, C., Bertelli, G. 1997, AJ, 114, 680

Duquennoy, A., Mayor, M. 1991, A\&A, 248, 485

Gallart, C., Freedman, W.L., Mateo, M., Chiosi, C., Thompson, I.B., Aparicio, A., Bertelli, G., Hodge, P., Lee, M.Y., Olsewszki, E.O., Saha, A., Stetson, P.B., Suntzeff, N. 1999, ApJ, 514 (2) (G99a)

Gallart, C., Freedman, W.L., Aparicio, A., Bertelli, G., Chiosi, C. 1999, in preparation (G99b)

Lee, M.G. Freedman, W.L., Mateo, M., Thompson, I., Roth, M., Ruiz, M.-T. 1993, AJ, 106, 1420

Knapp, G.R., Kerr, F.J., Bowers, P.F. 1978, AJ, 83, 228

Kroupa, P., Tout, C.A., Gilmore, G. 1993, MNRAS, 262, 545

Olszewski, E.O., Pryor, C., Armandroff, T.E. 1996, AJ, 111, 750

Stetson, P.B. 1994, PASP, 106, 250

\section{Discussion}

Zaritszky: Can you comment further on the relative contribution to $\chi^{2}$ from your various CMD boxes (i.e., does one box in particular dominate when $\chi^{2}$ starts deviating from 1)? Specifically, can you comment on which boxes are driving the large binary fractions?

Gallart: The boxes (usually several) that dominate the value of $\chi^{2}$ vary from one model to another, i.e., different models fail to reproduce different parts of 
the CMD. The boxes that produce large values of $\chi^{2}$ if we don't include binaries in the models are mainly those situated in the subgiant branch. That part of the CMD tends to be underpopulated if we don't include binaries.

Marconi: Do you try to compare also the luminosity functions derived from your "best models"?

Gallart: What we are doing in our comparison is, in fact, generalizing the luminosity-function method to a "luminosity-color-function" method. We require that the number of stars, both as a function of luminosity and color, agree with the observations. Therefore, this includes implicitly the comparison with luminosity functions.

Pritchet: Have you tried minimizing a functional other than $\chi^{2}$, for example, the sum of the absolute deviations of model and observations? This would be less sensitive to, for example, problems that might exist in the models.

Gallart: No, we haven't tried that in particular. Thanks for the suggestion.

Armandroff: I was wondering how much more information you would get if your photometry went deeper still? How would you change the way you do the analysis?

Gallart: Reaching the oldest main-sequence turnoffs gives you most of the information on age contained in the CMD. In this case, they are near the limit of our photometry, and this makes it more difficult to obtain reliable values for the star formation rate at old ages. However, the limited time resolution at old ages is, in any case, intrinsic to the fact that the stars formed, say, during the first third of the galaxy evolution (15-10 Gyr ago) are packed in a small area of the CMD. For this reason, the information in the horizontal branch is fundamental to study the old star formation history. If one reaches much deeper, as for example, in some of the HST CMDs of the Magellanic Clouds, one can start to investigate the initial mass function from the main-sequence stars. But I don't think I would change the method of analysis in any fundamental way.

Because I don't think that you would get much better age information going much deeper (but I would need to experiment with deeper data to be sure!), I think that the dSph satellites of the Milky Way and the Magellanic clouds can be studied better using large telescopes (4-m class and up) from the ground. With them, you can reach the critical depth in the CMD, and you benefit from a much larger area in the sky and therefore, a much better sampling of the stellar populations, with the possibility of analyzing spatial variations of the star formation history, for example. 